\title{
Modelling Macroscopic Phenomena with Cellular Automata and Parallel Genetic Algorithms: An Application to Lava Flows
}

\author{
Maria Vittoria Avolio ${ }^{1}$, Donato D'Ambrosio ${ }^{2}$, Salvatore Di Gregorio ${ }^{2}$, \\ Rocco Rongo ${ }^{1}$, William Spataro ${ }^{2}$, and Giuseppe A. Trunfio ${ }^{3}$ \\ ${ }^{1}$ Department of Earth Sciences, University of Calabria, 87036 Rende (CS), Italy \\ ${ }^{2}$ Department of Mathematics, University of Calabria, 87036 Rende (CS), Italy \\ ${ }^{3}$ DAP, University of Sassari, 07041 Alghero (SS), Italy \\ \{avoliomv, d.dambrosio, dig, spataro\}@unical.it, trunfio@uniss.it
}

\begin{abstract}
Forecasting through simulations the shape of lava invasions in a real topography represents a challenging problem, especially considering that the phenomenon usually evolves for a long time (e.g. from a few to hundreds of days) and on very large areas. In the latest years, Cellular Automata (CA) have been well recognized as a valid computational approach in lava flow modelling. In this paper we present some significant developments of SCIARA, a family of deterministic CA models of lava flows which are optimized for a specific scenario through the use of a parallel genetic algorithm. Following a calibration-validation approach, the model outcomes are compared with three real events of lava effusion.
\end{abstract}

Keywords: Modelling and Simulation, Cellular Automata, Lava flows.

\section{Introduction}

Lava flows consist of unconfined multiphase streams, the characteristics of which (e.g. temperature, rheologic parameters, velocity, phase state and shape) vary in space and time as a consequence of many interrelated physical and chemical phenomena (e.g. the flow dynamic itself, the loose of thermal energy through radiation, convecting air and conduction, the change in viscosity and the solidification processes). Thus, forecasting the shape of lava invasions in a real topography, given the lava effusion rate (i.e. the volumetric flux of lava from the vent), requires the ability to perform accurate simulations of a typical multiphysics system. Besides, considering that the phenomenon usually evolves for long time and on very large areas, standard approaches, such as those based on differential equations and related approximate techniques, result of difficult use.

On the other hand, it is well recognized that Cellular Automata (CA) can represent a valid alternative in lava flow modelling. A family of deterministic CA models specifically developed for simulating lava flows is SCIARA [12]3. The model, which is optimized for a specific scenario through a parallel Genetic Algorithm (GA), accounts for the relevant physical processes involved in the macroscopic phenomenon and enables for the fast production of accurate forecasting of lava invasions. In this paper some characteristics of the latest version, 
named SCIARA-fv, which introduces many model improvements and computational optimizations, is illustrated. Given the availability of historical data regarding various eruptive episodes of Mount Etna (Italy), which is the most active volcano in Europe, a cross-validation methodology is exploited for both the parameter calibration and assessment of the model reliability in that specific scenario. To this end, a new measure which takes into account simultaneously the spatial and temporal dimensions has been employed as fitness function for the evaluation of the simulation results with respect to the real events. A parallel Master-Slave GA is used for the model calibration in a training-case while the validation is carried out on a test set constituted by different real episodes.

\section{Model Specification}

SCIARA-fv is the latest release in a family of bi-dimensional CA models for lava flows simulation, based on hexagonal cells. The model follows the Macroscopic Cellular Automata (MCA) approach 4, where: $(i)$ the state of the cell is decomposed in substates, each one representing a particular feature of the phenomenon to be modelled; $(i i)$ the transition function is split in elementary processes, each one describing a particular aspect of the considered phenomenon. Moreover, some parameters are generally considered, which allow to "tune" the model for reproducing different dynamical behaviours. Finally, several external influences can be considered in order to model features which are not easy to be described in terms of local interactions. Even though principally derived from the SCIARA-hex1 version [3], SCIARA-fv embeds a better management of several aspects with respect to the original one, some of which will be described later. Formally, the model is defined as:

$$
\text { SCIARA-fv }=\langle R, L, X, Q, P, \lambda, \gamma\rangle
$$

where:

$-R$ is the set of hexagonal cells covering the finite region where the phenomenon evolves;

- $L \subset R$ specifies the lava source cells (i.e. vents);

- $X=\{$ Center, $N W, N E, E, S E, S W, W\}$ identifies the hexagonal pattern of cells that influence the cell state change (i.e. the cell itself, Center, and the North-West, North-East, East, South-East, South-West and West neighbors);

$-Q=Q_{a} \times Q_{t} \times Q_{T} \times Q_{f}^{6}$ is the finite set of states, considered as Cartesian product of "substates". Their meanings are: cell altitude, cell lava thickness, cell lava temperature, and outflows lava thickness (from the central cell toward the six adjacent cells), respectively. It is worth to observe that, in the model, the third dimension, the height, is included as a property of the state of the cell (i.e. a substate);

$-P=\left\{p_{s}, p_{T v}, p_{T s o l}, p_{a d v}, p_{a d s o l}, p_{c o o l}, p_{a}\right\}$ is the finite set of parameters (invariant in time and space), which affect the transition function (see Tab. 1 for the meanings of parameters in $P$ ); 
$-\lambda: Q^{7} \rightarrow Q$ is the cell deterministic transition function;

$-\gamma: Q_{t} \times \mathbb{N} \rightarrow Q_{t}$ specifies the emitted lava thickness from the source cells at each step $k \in \mathbb{N}$.

The main elementary processes which compose the transition function are outlined, in order of application, in the following subsections.

Lava Flows Computation. Lava rheological resistance is modelled in terms of an adherence effect, measured by $\nu$, which represents the amount of lava (i.e. thickness) that cannot flow out of a cell towards any neighboring ones. In particular, since lava rheological resistance increases as temperature decreases, the model is expressed by means of the inverse exponential function [5]:

$$
\nu=k_{1} e^{k_{2} T}
$$

where $T \in Q_{T}$ is the current lava temperature, while $k_{1}$ and $k_{2}$ are parameters depending on lava rheological properties [6]. The values of $k_{1}$ and $k_{2}$ are simply obtained by solving the system of equations:

$$
\left\{\begin{array}{l}
p_{a d v}=k_{1} e^{-k_{2} p_{T v}} \\
p_{\text {adsol }}=k_{1} e^{-k_{2} p_{T s o l}}
\end{array}\right.
$$

Since the third spatial dimension, i.e. the height, is included in the model as a substate, cell outflows can be computed by the algorithm for the minimization of differences described in [4. The minimization algorithm, which is based on the iterative fulfilment of the hydrostatic equilibrium principle through the minimization of the differences in height, guarantees the mass conservation.

Let $s[k]$ indicate the current value of the substate $s$ for the $k$-th cell of the neighborhood, with $k=0$ for the cell under consideration. Let also $a \in Q_{a}$ and $t \in Q_{t}$ be the cell altitude and cell lava thickness, respectively. In order to compute lava outflows from the central cell towards its neighboring ones, the minimisation algorithm is applied to the following quantities: the unmovable part $u[0]=a[0]+\nu$ and the mobile part $m[0]=t[0]-\nu$ (i.e. the only part which can be distributed to the adjacent cells). Let $\phi[x, y]$ denote the flow from cell $x$ to cell $y$, thus $m[0]$ can be written as:

$$
m[0]=\sum_{i=0}^{\sharp X} \phi[0, i]
$$

Table 1. The parameters used in SCIARA-fv model

\begin{tabular}{cl}
\hline Parameter & \multicolumn{1}{c}{ Definition } \\
\hline$p_{s}$ & time corresponding to a CA step \\
$p_{T v}$ & lava temperature at the vent \\
$p_{T s o l}$ & lava temperature at solidification \\
$p_{a d v}$ & lava adherence at the vent \\
$p_{a d s o l}$ & lava adherence at solidification \\
$p_{\text {cool }}$ & the cooling parameter \\
$p_{a}$ & cell apothem \\
\hline
\end{tabular}


where $\phi[0,0]$ is the part which is not distributed, and $\sharp X$ is the number of cells belonging to the neighborhood $X$. During the minimization, the quantities in the adjacent cells, $u[i]=a[i]+t[i](i=1,2, \ldots, \sharp X)$ are considered unmovable. Let $c[i]=u[i]+\phi[0, i] \forall i$ be the new quantity content in the $i$-th neighboring cell after the distribution and let $c_{\min }$ be the minimum value of $c[i] \forall i$. The outflows are computed in order to minimise the expression $\sum_{i=0}^{\sharp X}\left(c[i]-c_{\min }\right)$. In 4 it is shown that the simultaneous application of the minimization principle to each cell gives rise to the global equilibrium of the system.

Thermal Exchanges and Lava Solidification. It is assumed that a lava flow can be treated as if thermally well mixed, so that each cell has a temperature field constant in space. Besides, only thermal energy loss due to lava surface irradiation is considered. Although such a cooling model may not always be realistic, it does yield a characteristic cooling time scale comparable to representative emplacement times of some important typology of lava flows.

Under this hypotheses, the new cell temperature $T_{t+\Delta t}$ is determined in two phases. In the first phase, which accounts for the energy exchange directly related to the mass exchange, the cell temperature $T_{a v g}$ is obtained as weighted average of residual lava inside the cell and lava inflows from neighboring ones:

$$
T_{\text {avg }}=\frac{t_{r} T_{t}[0]+\sum_{i=1}^{6} \phi[i, 0] T_{t}[i]}{t_{r}+\sum_{i=1}^{6} \phi[i, 0]}
$$

where $t_{r} \in Q_{t}$ is the residual lava thickness inside the central cell after the outflows distribution, $T_{t}[k] \in Q_{T}$ is the lava temperature at the time step $t$ for the $k$-th cell of the neighborhood (i.e. $k=0$ for the cell under consideration) and $\phi[i, 0]$ the lava inflow from the $i$-th neighboring cell. Note that $\phi[i, 0]$ corresponds to the lava outflow from the $i$-th neighboring cell towards the central one, computed by means of the minimisation algorithm.

The final step updates the temperature given by (5) considering thermal energy loss due to lava surface irradiation, which can be expressed by the StefanBoltzmann equation. In particular, starting from a temperature $T_{t}$ a good approximation of the temperature $T_{t+\Delta t}$ is given by [6]:

$$
T_{t+\Delta t}=T_{t}\left(1+\frac{3 T_{t}^{3} \varepsilon \sigma A \Delta t}{\rho c V}\right)^{-\frac{1}{3}}
$$

where $\rho$ is the lava density, $c$ the specific heat, $V$ the volume, $\sigma$ the StephanBoltzmann constant, $T_{t}$ the absolute temperature at time $t, A$ is the air exposed area of the cell, $\varepsilon$ is the surface emissivity and $\Delta t$ is the step duration of the CA. Thus, according to (6), the temperature $T_{a v g}$ computed in (5) is updated through the following relation:

$$
T_{t+\Delta t}[0]=T_{a v g}\left(1+\frac{p_{\text {cool }} T_{a v g}^{3} A p_{s}}{V}\right)^{-\frac{1}{3}}
$$

where $p_{\text {cool }}$ accounts for the relevant lava's physical properties and $p_{s}=\Delta t$ (see Tab. 1). It is worth to note that the parameter $p_{\text {cool }}$, the measurement 
of which would be quite difficult, is directly determined during the calibration process exploiting the availability of real spatio-temporal data regarding past events of the effusion of a specific kind of lava. Finally, if the temperature drops below the threshold $p_{T s o l}$, the lava solidifies, that is, its thickness is added to the geographical altitude of the cell and the lava thickness is reset to zero.

\section{Model Calibration}

Once that a MCA model has been defined, two stages are needed to assess its reliability: the calibration and validation phases. The former searches a set of parameters able to adequately reproduce a specific real case; the latter tests the model on a sufficient number of cases (which must be different of those considered in the calibration phase, though similar in terms of physical and geological properties), permitting to give a final response on the model goodness.

The calibration phase is performed by means of a GA similar to that applied in 7, where parameters to be optimised were encoded as bit strings. Moreover, the GA adopts a steady-state and elitist model, so that at each step only the worst individuals are replaced. The remaining ones, required to form the new population, are copied from the old one, choosing the best. In order to select the individuals to be reproduced, the "binary-tournament without replacement" selection operator was utilised. It consists of a series of "tournaments" in which two individuals are selected at random, and the winner is chosen according to a prefixed probability (0.6 in our case), which must be set greater for the fittest individual. Moreover, as the variation without replacement scheme was adopted, individuals cannot be selected more than once. Employed genetic operators are classic Holland's crossover and mutation with probability of 1.0 and 2/44, respectively. In particular, the latter probability implies, on an average, two bits mutated for each individual, as the genotype length was exactly 44 (see Tab. 22). The number of individuals forming the initial population was set to 256, while the number of individuals to be replaced at each GA step was set to 16 .

One of the most important aspects of the calibration phase is the definition of the fitness function, i.e. the function which measures the level of agreement between the real event and the simulated one. In order to the model to well describe the overall spatio-temporal dynamics of the system, the simulation should be able to produce outcomes which correspond with the real phenomenon in terms of its relevant characteristics (e.g. areal extension and shape, lava thickness and temperature, invasion velocity) at some specific instants $\left\{t_{1}, \ldots t_{k}\right\} \in[0, \tau]$, being $\tau$ the duration of the real event. Unfortunately, complete mapping of lava characteristics corresponding to instants $t<\tau$ are rarely surveyed by geologists, while only the final shape of invasion is usually available. On the other hand, for risk analysis purposes, the correspondence between real and simulated lava shapes in the instant $t=\tau$ in which the real phenomenon reaches a steady-state condition, is of particular interest. For the above reasons, the fitness function to be maximised was defined in such a way to combine the two objectives as:

$$
\Theta=\sqrt{\Omega_{\tau} \Omega_{\tau+\Delta \tau}}
$$


Table 2. The best set of SCIARA-fv parameters as obtained through calibration phase

\begin{tabular}{cccc}
\hline Parameter & Explored range & Encoding bits & Best value \\
\hline$p_{s}$ & {$[60,180]$} & 8 & $155.29 \mathrm{~s}$ \\
$p_{T v}$ & - & - & $1373 \mathrm{~K}$ \\
$p_{T \text { sol }}$ & {$[1123,1173]$} & 8 & $1165.35 \mathrm{~K}$ \\
$p_{\text {adv }}$ & {$[0.1,2.0]$} & 4 & $0.7 \mathrm{~m}$ \\
$p_{\text {adsol }}$ & {$[6.0,30.0]$} & 6 & $12 \mathrm{~m}$ \\
$p_{\text {cool }}$ & {$\left[10^{-16}, 10^{-13}\right]$} & 16 & $2.9 \times 10^{-14} \mathrm{~m}^{3} \mathrm{~K}^{-3}$ \\
$p_{a}$ & - & - & $5 \mathrm{~m}$ \\
\hline
\end{tabular}

where $\Omega \in[0,1]$ is a suitable measure of the spatial agreement between real and simulated lava invasions, with $\Omega=1$ in case of perfect overlapping, while $\Delta \tau$ is a small surplus time. When $\Theta=1$, then real and simulated events perfectly overlap with the further condition $\Omega_{\tau}=\Omega_{\tau+\Delta \tau}$, meaning that the simulation reaches the steady-state exactly at the same time as the real event does. As regards function $\Omega$ in (8), it was defined as:

$$
\Omega_{t}=\sqrt{\frac{m(R \cap S)}{m(R \cup S)}}
$$

where $t$ is the instant of evaluation, $R$ and $S$ represent the sets of cells affected by the real and simulated event, respectively, while $m(A)$ denotes the measure of the set $A$. Note that $\Omega \in[0,1]$ and its value is 0 if the real and simulated events are completely disjoint, being $m(R \cap S)=0$, while it is 1 in case of perfect overlap, being $m(R \cap S)=m(R \cup S)$.

\section{A Case of Study}

At 3.00 AM on July 18-th, 2001, an eruption started from the fracture of Mount Calcarazzi, on the southern flank of Mt Etna (Sicily, Italy), at $2100 \mathrm{~m}$ a.s.l. The event was fed by a medium lava flow rate (about $7 \mathrm{~m}^{3} / \mathrm{s}$ ) and, due to the steep descent of the terrain in that area, pointed southwards creating the main danger for the towns of Nicolosi and Belpasso. After 10 days of activity, it reached its maximum extension, which was almost $6 \mathrm{Km}$ in terms of run-out. Such event was chosen as the reference case for the calibration phase, as it was considered sufficiently representative of Etnean lava flows and even characterised by a relative brief duration. In particular, the second feature allowed to execute the elevated number of simulations required by the GA in a reasonable amount of time. Moreover, to further speedup the experiments execution, a Master-Slave parallel GA version was considered, instead of the sequential one, which simply split the individuals' fitness evaluation over the available "slave" processors, while the GA steps are managed by the "master" one. It represents the simplest example of parallel GA [8]. Accordingly, calibration was performed on a Nec TX7 NUMA machine, composed by 4 quadri-processors Itanium class nodes, with an overall RAM memory of $32 \mathrm{~GB}$ and a peak performance of 64 GFLOPS. 

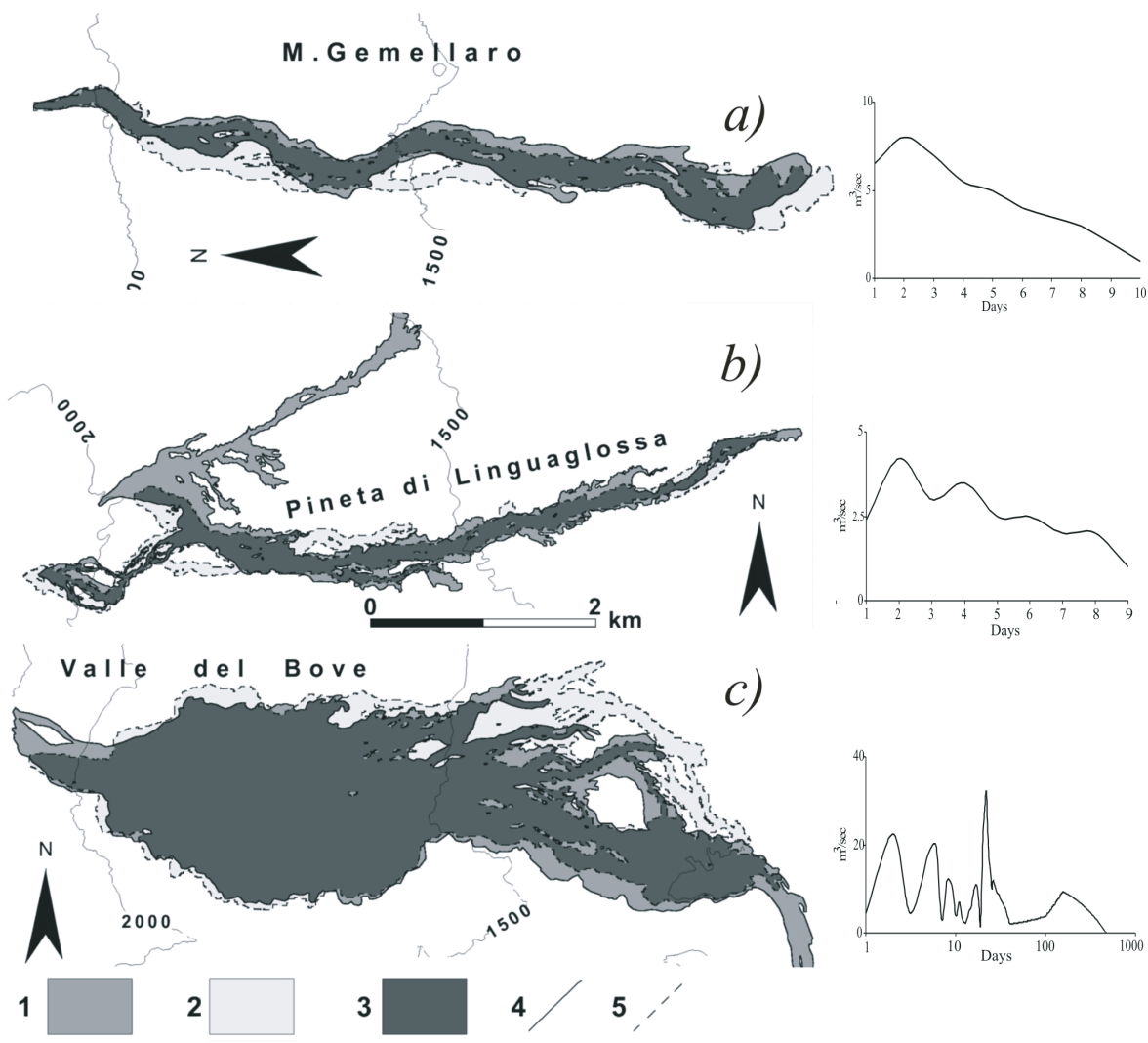

Fig. 1. Comparison between real and simulated events (charts report the lava emission rate). Keys: 1) Area affected by the real event but missed by the simulation; 2) Area incorrectly forecasted as invaded by lava; 3) Area correctly forecasted as invaded by lava; 4) Limits of real event, 5) Limits of simulated event.

On the basis of previous empirical attempts, ranges within which the values of the CA parameters are allowed to vary were individuated in order to define the GA search space (see Tab. 2), and a set of 10 experiments iterated for 100 steps. As regards the fitness function, $\Omega_{\tau}$ was evaluated after $\tau=10$ days (which corresponds to the duration of the real event), while $\Delta \tau$ was set to 3 days. Results of the calibration phase are reported in Tab. 2, Note that parameter $p_{T v}$ was set to a prefixed value, which corresponds to the typical temperature of Etnean lava flows at vents. Parameter $p_{a}$ was also prefixed, as it was imposed by the detail of the considered topographic data. The parameter set listed in Tab. 2 allowed to reproduce the considered 2001 Nicolosi Etnean lava flow (see Fig. 1 , case $a$ ), giving rise to a fitness $\Theta=0.72$, which corresponds to $\Omega_{\tau}=0.74$ in terms of areal comparison. Such values indicate a good performance of the model considering the significant uncertainties which affect both the measured effusion rate and the available topography data (while the GA proved to be able 
to explore quite well the parameter space, since in a calibration test against the outcomes of the model itself it provided a solution with $\Omega_{\tau} \approx 0.95$ ). Besides, as it can be observed in Fig. 1 a fitness value of 0.72 can be considered as satisfactory from a risk assessment and hazard mapping point of view, since the adopted measure is fairly severe in terms of shape concordance.

The validation phase was carried out by testing the obtained parameters to other real cases of study: the 2002 Linguaglossa and the 1991 - 93 Valle del Bove events, both regarding Mt Etna (Sicily). The first lasted 9 days, the second 473 days. Results are graphically illustrated in Fig.1, case b) and c), respectively with the corresponding emission rates. As expected, the best set of parameters (see Tab. 2) permitted a satisfactorily reproduction of the considered phenomena: in quantitative terms, the obtained $\Omega_{\tau}$ values were 0.71 and 0.85 , respectively.

\section{Conclusions}

Simulating real lava invasions for hazard management requires models characterized by high reliability, robustness and low computational cost. Latest results, reported in this paper, confirm that the macroscopic approach based on CA and evolutionary computation can provide such characteristics. Nevertheless, further improvements could be achieved overcoming some of the simplified assumptions of the presented model. Future research work will be addressed in this direction.

\section{References}

1. Crisci, G., Di Gregorio, S., Ranieri, G.: A cellular space model of basaltic lava flow. In: Proceedings International AMSE Conference Modelling \& Simulation (Paris, France, Jul.1-3). (1982) 65-67

2. Barca, D., Crisci, G., Di Gregorio, S., Nicoletta, F.: Cellular automata for simulating lava flows: A method and examples of the Etnean eruptions. Transport Theory and Statistical Physics 23 (1994) 195-232

3. Crisci, G., Rongo, R., Di Gregorio, S., Spataro, W.: The simulation model sciara: the 1991 and 2001 lava flows at mount Etna. Journal of Volcanology and Geothermal Research 132 (2004) 253-267

4. Di Gregorio, S., Serra, R.: An empirical method for modelling and simulating some complex macroscopic phenomena by cellular automata. Future Generation Computer Systems 16 (1999) 259-271

5. McBirney, A., Murase, T.: Rheological properties of magmas. Annual Review of Earth Planetary Sciences 12 (1984) 337-357

6. Park, S., Iversen, J.: Dynamics of lava flow: Thickness growth characteristics of steady two-dimensional flows. Geophysical Research Letters 11 (1984) 641-644

7. Spataro, W., D'Ambrosio, D., Rongo, R., Trunfio, G.A.: An evolutionary approach for modelling lava flows through cellular automata. In Sloot, P.M.A., Chopard, B., Hoekstra, A.G., eds.: LNCS - ACRI. (2004) 725-734

8. Cantu-Paz, E.: Efficient and Accurate Parallel Genetic Algorithms. Kluwer Academic Publishers, Norwell, MA, USA (2000) 